\title{
Outflows and the Disk-Halo Connection in Galaxies
}

\author{
Judith A. Irwin \\ Queen's University, Kingston, Ontario, Canada, K7L $3 N 6$
}

\begin{abstract}
There is now abundant evidence for high latitude gas in the Milky Way and external edge-on galaxies. The halo gas is seen in various ISM tracers, including $\mathrm{HI}, \mathrm{H} \alpha$, and radio continuum, and shows considerable structure, including discrete features which span the disk-halo interface. While some correlation with underlying star formation activity has been observed, several energy discrepancies are outlined which suggest that conventional processes related to massive star formation are not sufficient to account for the observed emission. Indeed, new sensitive data are showing that high latitude gas may be a common characteristic of all galaxies, not just those with high star formation rates.
\end{abstract}

\section{The Milky Way}

\subsection{Early Observations of High-Latitude Gas}

It has been over 40 years since high latitude gas was first suspected in the Milky Way. As early as 1956, Spitzer stated, "gas may actually be present far from the galactic plane." A few years later, Munch \& Zirin (1961) claimed, "some gas clouds probably exist at $\mathrm{z}=1 \mathrm{kpc}$." And from Oort, in 1962, "well outside the real disk one still finds neutral hydrogen with an average density of between 5 and 10 per cent of the density in the plane." Remarkably, Oort's early estimate for extra-planar neutral hydrogen (HI) is still in rough agreement with modern values (Lockman 1991).

\subsection{Neutral Hydrogen}

The thickness of the HI layer in the Milky Way depends on galactocentric radius, with the thinnest region in the inner few kpc and flaring becoming more obvious beyond the solar circle. Between radii of 4 and $8 \mathrm{kpc}$, the mean $\mathrm{z}$ distribution can be described by a smooth density function which is a combination of several components with FWHM values up to 530 pc (Dickey \& Lockman 1990). The high latitude ( $>500 \mathrm{pc}$ ) gas has become known as the Lockman layer. More recently, faint wings have been identified on HI line profiles in the Leiden-Dwingeloo Survey (LDS, Hartmann \& Burton 1997) implying the presence of high velocity dispersion $\left(\sim 60 \mathrm{~km} \mathrm{~s}^{-1}\right)$ gas. If this gas is in hydrostatic equilibrium, then a much larger scale height $(\sim 4.4 \mathrm{kpc})$ is implied (Kalberla et al. 1998).

While the average properties of the gas may be described by smooth functions, these mask the beautiful complexity and numerous discrete features which 


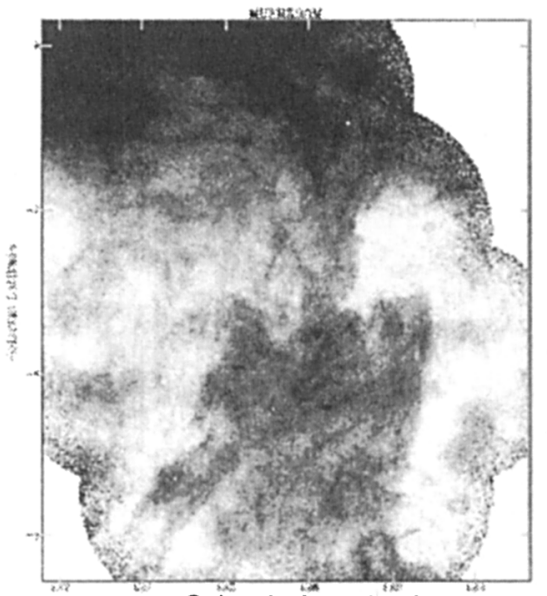

Galactic Longitude

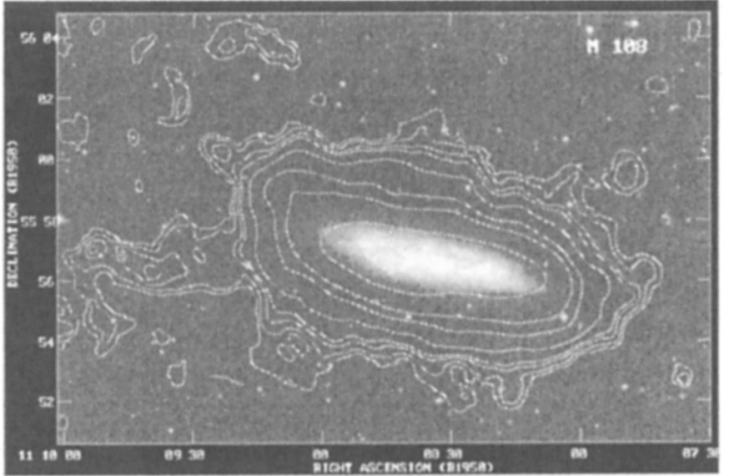

Figure 1. Examples of HI disk-halo features on very different scales. Left: An inverted mushroom-shaped HI worm in the Milky Way (few hundred pc, English et al. 2000). Right: Total intensity image of M 108 showing numerous extensions, especially to the east, and loops or partial loops, especially to the south (few kpc, King \& Irwin 1997).

are visible in any field of the LDS and continue to be revealed in the on-going higher resolution Canadian Galactic Plane Survey (Fig. 1, Left).

Such complexity was already hinted at in earlier surveys, however. Heiles $(1979,1984)$, for example, focussed attention on disk-halo dynamics by identifying 105 shells and shell-like objects in the Galaxy. Some of these show evidence for expansion, though typically only half-shells are observed. The largest shells have radii of $1.2 \mathrm{kpc}$, masses of $10^{7} \mathrm{M}_{\odot}$ and require input energies of $6 \times 10^{53}$ ergs. With a single supernova (SN) contributing a kinetic energy of $10^{51}$ ergs, the inferred requirement of $\sim 600$ SNe has been considered prohibitively large for shell formation. These input energies were computed assuming instantaneous deposition of energy. While a more modern approach is to allow energy input over some time frame appropriate to the parent cluster and to include stellar winds, the quantitative results are not dissimilar. Thus there is an energy problem for the largest shells.

The front-runner for alternative supershell generation is impacting external clouds. If the supershells are produced by external clouds "splashing" onto the disk, the available energy can be much higher, being limited by the mass and velocity of the incoming cloud itself. This hypothesis has gained support from observations of high velocity clouds near our own Galaxy and possible connections with features such as the anticenter shell (Mirabel \& Morras 1990), the North Celestial Pole Loop (Meyerdierks 1992) and Gould's Belt (Comerón \& Torra 1994). Impactors have also been implicated in external galaxies such as M 101 (van der Hulst \& Sancisi 1988) and NGC 4631 (Rand \& Stone 1996). For recent theoretical models, see Comerón and Torra (1992) and Santillan et al. (1999). 
Aside from shells, Heiles also identified numerous Galactic "worms" which "crawl" out of the plane (Fig. 1, Left) and are located mostly in the inner Galaxy. The worms, if open-topped, are often referred to as Galactic "chimneys", as described by Norman and Ikeuchi (1989), though there may be little or no evidence for upwardly moving hot gas in their interiors. A textbook chimney will have a single stellar cluster at its base in which SNe open up a channel into the halo region above. The remaining hot stars then ionize the inner walls of the chimney, provided the ionizing photons can initially escape from the gas in the cluster region at the base. Though real worms are rather more complex, there does appear to be a correlation between ionized worm walls and the presence of stellar clusters (Heiles, Reach, \& Koo 1996).

\subsection{Diffuse Ionized Gas}

Ionized $10^{4} \mathrm{~K}$ gas, called the Reynolds layer in our own Galaxy (also known as Diffuse Ionized Gas, DIG) also exists well above the Galactic disk. The scale height of this component is determined from pulsar dispersion measures over various sight lines, resulting in the functional form, $\mathrm{n}_{e}=0.024 \exp (-\mathrm{z} / 950 \mathrm{pc})$ $\mathrm{cm}^{-3}$ within $3 \mathrm{kpc}$ of the sun (Reynolds 1997). A very high energy input rate, i.e. $10^{-4}$ ergs s${ }^{-1}$ per $\mathrm{cm}^{2}$ of Galactic disk is required to keep this layer ionized. This rate corresponds to $100 \%$ of the power from all SNe or $16 \%$ of all OB star ionizing radiation. The energy input from other sources, such as white dwarfs or cosmic rays, is lower. Since, clearly, some SN energy must go elsewhere, most authors have concentrated on ionization from the OB star population in the disk as the source. This, of course, requires that a significant fraction of the $\mathrm{OB}$ ionizing radiation both escapes from density bounded HII regions and also reaches the heights observed, presumably through chimneys, before being absorbed. Some of the observed line ratios (e.g. [SII] $\lambda 6717 / \mathrm{H} \alpha,[\mathrm{NII}] \lambda 6583 / \mathrm{H} \alpha$, [OIII] $\lambda 5007 / \mathrm{H} \alpha)$ are consistent with ionization by such a dilute radiation field. However, the fact that He ionization is lower than expected implies that the mean ionizing spectral type is later than 08. Since only $22 \%$ of the ionizing flux is emitted from such stars, this means that almost all of their ionizing radiation must escape into the halo to provide the required energy (Domgörgen 1997), a rather unlikely scenario. Thus an energy problem also exists for the DIG.

\subsection{Other Components and Identifying High Latitude Gas}

Large scale heights have also been observed in other ISM components. Among these are X-rays at $4.4 \mathrm{kpc}$ (Pietz et al. 1998), highly ionized species at $4 \mathrm{kpc}$ (Savage, Sembach, \& Lu 1997), and $408 \mathrm{MHz}$ radio continuum at a variety of scales heights from $1.5 \mathrm{kpc}$ to $4.8 \mathrm{kpc}$ (see Kalberla \& Kerp 1998).

For continuum emission, it is clearly difficult to find the scale height since a model is required. For line emission like HI, an assumption that the halo gas is corotating with the disk is also required. While there is no strong evidence against this, in at least one external galaxy, NGC 891, the HI halo has been shown to lag behind the rotation of the disk (Swaters, Sancisi, \& van der Hulst 1997). Moreover, for dynamical features which may, in fact, be half-shells seen over $\sim 25 \mathrm{~km} \mathrm{~s}^{-1}$, one must be very careful in determining the central velocity in order to fix a kinematical distance. The large sizes of some of these features 
can also make it challenging to identify them at all (cf. the mushroom of Fig. 1 which spans 3 degrees of sky).

A good way to overcome these problems is to observe external galaxies. In edge-on galaxies, in particular, there is no ambiguity as to whether the gas is extra-planar (though velocity information becomes more problematic). If nearby galaxies are chosen, available instruments can also resolve the supershells, both in velocity and spatially. Note, however, that the largest features will still be preferentially selected. Thus, it is not yet straightforward to compare the features seen in external galaxies with those seen in the Milky Way. Nevertheless, as stated by Heiles (1984), "The study of shells in external galaxies probably offers the best way to understand their origins."

\section{External Edge-on Galaxies}

\subsection{Diffuse Ionized Gas}

Extraplanar DIG has been observed in a number of external galaxies, though in general it appears to be rare (Rand 1996). A nice illustration of extraplanar $\mathrm{H} \alpha$ emission can be found in Hoopes, Walterbos, \& Rand (1999). There is good evidence that this emission is related to the underlying star forming disk. For example, in NGC 891, discrete filaments appear connected to specific star forming regions in the disk. There also appears to be a correlation between the in-disk tracers of star formation such as [CII] $158 \mu \mathrm{m}$ emission and halo $\mathrm{H} \alpha$ (see Rand 1997). On the other hand, the ionization energy deficit appears to be even more extreme in external galaxies; perhaps $30-50 \%$ of all ionizing photons are required (see Hoopes et al. 1999 and references therein). Thus, processes related to massive star formation do not alone appear to be sufficient to account for the ionized halos.

\subsection{Radio Halos}

The most thorough search for radio continuum halos in edge-on systems was carried out by Hummel, Beck, and Dettmar (1991) who surveyed 181 galaxies at $\lambda 6 \mathrm{~cm}$ using the Very Large Array (VLA) in its D configuration. These galaxies were small (< about 4 arcmin) and were observed for 15 minutes each. The result was that only 10 galaxies, or $5.5 \%$ of the sample, showed evidence for high latitude radio continuum.

In spite of the low detection rate, a few galaxies, such as NGC 4631, were well known to have very extensive radio halos. Dahlem, Lisenfeld, \& Golla (1995) looked at 8 external galaxies and found an approximate relation between the existence of radio halos and the underlying input energy rate per unit area from supernovae. An exception was NGC 4631 which has an exceptionally strong halo for its underlying supernova rate. They also noted a spatial correlation between the radio halos and $\mathrm{H} \alpha$ disk distributions for NGC 891 and NGC 4631.

Such studies are particularly challenging, given that so few galaxies show high latitude $\mathrm{H} \alpha$ or radio continuum, the available data have varying noise levels, and the halo emission is both weak and complex. The latter point has made it difficult to find a quantifiable measure of halo strength, so halo morphology has been used instead. A good illustration of the difficulties is given by NGC 5775 in 

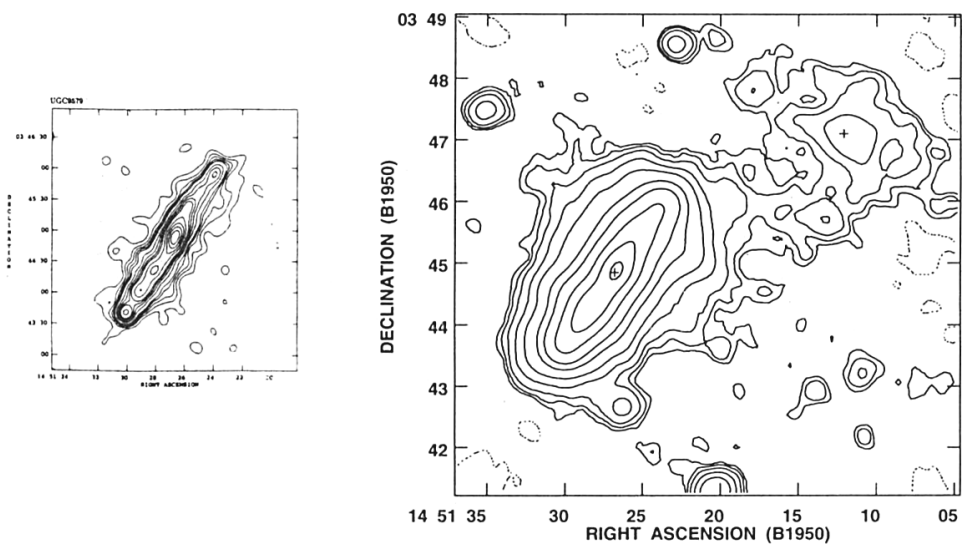

Figure 2. Radio continuum images of NGC 5775, plotted to the same linear scale, illustrating how a morphological description of the radio halo is clearly a function of frequency, sensitivity and resolution. Left: $\lambda 6 \mathrm{~cm}$ image from Hummel et al. (1991) using the VLA in its D configuration for 15 minutes. Right: $\lambda 20 \mathrm{~cm}$ image adapted from Duric et al. (1997) combining data from the B, C, and D configurations of the VLA over a total of 8 hours. A companion galaxy is centered at the cross to the north-west.

which its halo might only be described as "filamentary" from $\lambda 6 \mathrm{~cm}$ observations (Fig. 2, Left), but might be considered a strong halo from multi-array $\lambda 20 \mathrm{~cm}$ observations (Fig. 2, Right). What is needed is a sensitive survey in which a large number of radio halos can be characterized in a more quantitative fashion.

Recently, Irwin, English and Sorathia (1999) observed 16 edge-on galaxies at $\lambda 20 \mathrm{~cm}$ and a subset of these at $\lambda 6 \mathrm{~cm}$ using the VLA in C and D configurations. While still not quantifying the halos, they used several criteria to apply a rank indicating the strength and prevalence of the extra-planar emission. They found high latitude radio continuum in 15 of the 16 galaxies, a surprisingly high detection rate, considering that these galaxies were not, in general, starbursts, and suggesting that radio halos are common. For some of the galaxies which were not well resolved, follow-up A array observations have confirmed the extraplanar emission (Irwin, Saikia \& English 2000). From these data, no correlation was found between the prevalence of radio halos and the underlying supernova rate per unit area. A possible explanation is the respective timescales. For example, the lifetimes of the halo particles, assuming minimum energy, are typically $10^{8} \mathrm{yr}$, whereas stellar clusters and starbursts have lifetimes of a few $10^{7}$ yr. Thus radio halos may be measuring an integration of star formation rate over a longer time period than is indicated by current star formation activity in the disk. Alternatively, some other process, aside from or in addition to star formation may be producing the halos.

\subsection{Neutral Hydrogen}

Neutral hydrogen supershells have now been observed in a number of external galaxies but expansion signatures are difficult to observe, especially if only parts of shells are present. The galaxy, M 108, is a case in which there is evidence 
for expanding half-shells, with two features occurring at both the east and west ends of the disk, though other shell-like features are also seen throughout the disk (Fig. 1). The eastern shell in M 108, requires an input energy of $\sim 10^{56}$ ergs, or the correlated input of $\sim 170,000$ supernovae, if indeed they are the cause. Follow-up continuum observations failed to find any compact sources which might reasonably account for the shells, however (Irwin et al. 2000). This discrepancy is more difficult to explain by appealing to timescales (as above) because the dynamical lifetime of the shells is $\sim 10^{7}$ yr which is comparable to a starbursting or stellar cluster lifetime. Similar arguments hold for supershells observed in other galaxies, though few have been specifically probed for massive star forming regions.

Again, the alternative is to appeal to external cloud impacts. However, at least 2 of the supershell galaxies (NGC 3044, Lee \& Irwin 1998, and M 108) have no known companion. Since each galaxy shows evidence for several large shells of similar ages and with input energies up to $10^{56} \mathrm{ergs}$, it would be necessary to postulate that a "rain" of infalling clouds has occurred. Since impacting timescales (i.e. dynamical timescales) are of order $10^{8} \mathrm{yr}$, some of these clouds should still be present around the parent galaxy. Clouds which could have produced these large shells, however, would have been seen in the data (see King \& Irwin 1997, Lee \& Irwin 1997). (An exception would be if the clouds had very narrow line widths or low fractal dimensions in which case they could have been missed due to dilution factors.) Thus, an explanation in terms of impacting clouds is not entirely satisfactory either.

\section{Summary and Consequences}

In summary, there is some evidence for a correlation between extraplanar gas and in-disk star formation. However, various energy discrepancies, including the ionization of the DIG and the input energies for supershells, both in our own and other galaxies, suggest that conventional processes related to massive star formation are not, alone, sufficient to account for the observed emission. Given that many HI shells show no evidence for star forming regions within them, Efremov, Elmegreen, \& Hodge (1998) and Loeb \& Perna (1998) suggest that HI shells are produced by gamma ray bursts. Parker instabilities have also been proposed (Kamaya et al. 1996). It would appear that an important driver (and energy source) is still missing, and one would expect there to be some relation between this driver and star formation. This could mean that star formation is a consequence, rather than a cause of the high latitude gas. For example, if magnetic fields exported gas to high latitudes, such gas might then slide down the field lines, producing star forming regions. Or a rain of (say) fractal clouds might trigger star formation as they impact and compress in-disk gas.

Finally, while the root cause of the extra-planar gas is still debatable, the consequences of the outflow are no less interesting. Reverting to the star formation models, various estimates have been made for the mass loss rate from the disk, for example, 0.1 to $3 \mathrm{M}_{\odot} \mathrm{yr}^{-1}$ (Norman \& Ikeuchi 1989) 1 to $5 \mathrm{M}_{\odot}$ $\mathrm{yr}^{-1}$ (Breitschwerdt, Völk, \& McKenzie 1991, Breitschwerdt, McKenzie, \& Völk 1993), 1.2 to $300 \mathrm{M}_{\odot} \mathrm{yr}^{-1}$ (Heckman, Armus, \& Miley 1990), and 1 to $3 \mathrm{M}_{\odot}$ $\mathrm{yr}^{-1}$ (Suchkov et al. 1994). If, typically, $2 \mathrm{M}_{\odot} \mathrm{yr}^{-1}$ leave the disk, then over 
the galaxy's lifetime, the entire ISM could be cycled through the halo. If some of the gas escapes into intergalactic space, then a severe depletion of the ISM may occur, depending on what fraction of the gas is involved and the timescale over which this occurs. This is one area where the GMRT could be especially useful, i.e. in a search for very broad scale radio halos around galaxies which might distinguish between circulation or outflow. In either case, outflows can impose a significant perturbation on the evolution of the galaxy and must be thoroughly characterized if we are to understand this process.

\section{References}

Breitschwerdt, D., Völk, H. J., \& McKenzie, J. F. 1991, A\&A, 245, 79

Breitschwerdt, D., McKenzie, J. F., \& Völk, H. J. 1991, A\&A, 269, 54

Comerón, F., \& Torra, J. 1992, A\&A, 261, 94

Comerón, F., \& Torra, J. 1994, A\&A, 281, 35

Dahlem, M., Lisenfeld, U., \& Golla, G. 1995, ApJ, 444, 119

Dickey, J., \& Lockman, F. 1990, ARA\&A, 28, 215

Domgörgen, H. 1997, in The Physics of Galactic Halos, ed. H. Lesch, R.-J. Dettmar, U. Mebold, \& R. Schlickeiser (Berlin: Academie Verlag), 67

Duric, N., Irwin, J., \& Bloemen, H. 1998, A\&A, 331,428

Efremov, Y. N., Elmegreen, B. G., \& Hodge, P. W. 1998, ApJ, 501, L163

English, J., Taylor, A. R., Maschchenko, S. Y., Irwin, J. A., Basu, S., \& Johnstone, D. 2000 , in press

Hartmann, D., \& Burton, W. B. 1997, Atlas of Galactic Neutral Hydrogen (Cambridge: Cambridge University Press)

Heckman, T. M., Armus, L., \& Miley, G. K. 1990, ApJS, 74, 833

Heiles, C. 1979, ApJ, 229, 533

Heiles, C. 1984, ApJS, 55, 585

Heiles, C., Reach, W. T., \& Koo, B.-C. 1996, ApJ, 466, 191

Hoopes, C. G., Walterbos, R. A. M., \& Rand, R. J. 1999, ApJ, 522, 669

Hummel, E., Beck, R., \& Dettmar, R.-J. 1991, A\&AS, 87, 309

Irwin, J. A., English, J., \& Sorathia, B. 1999, AJ, 117, 2101

Irwin, J. A., Saikia, D. J., \& English, J. 2000, AJ, in press

Kalberla, P. M. W., \& Kerp, J. 1998, A\&A, 339, 745

Kalberla, P. M. W., Westphalen, G., Mebold, U. Hartmann, D., \& Burton, W. B. $1998, A \& A, 332,61$

Kamaya, H., Mineshige, S., Shibata, K., Matsumoto, R. 1996, ApJ, 458,25

King, D. L., \& Irwin, J. A. 1997, NewA, 2, 251

Lee, S.-W., \& Irwin, J. A. 1997, ApJ, 490, 247

Lockman, F. 1991, in IAU Symp. 144, The Interstellar Disk-Halo Connection in Galaxies, ed. H. Bloemen (Dordrecht: Kluwer), 15

Loeb, A., \& Perna, R. 1998,ApJ, 503, 35

Meyerdierks, H., 1992, A\&A, 253, 515 
Mirabel, I. F., \& Morras; R. 1990, ApJ, 356, 130

Munch, G., \& Zirin, H. 1961, ApJ, 133, 11

Norman, C. A., \& Ikeuchi, S. 1989, ApJ, 345, 372

Oort, J. H. 1962, in The Distribution and Motion of Interstellar Matter in Galaxies, ed. L. Woltjer (New York: Benjamin), 3 and 71

Pietz, J., Kerp, J., Kalberla, P. M. W., Burton, W. B., Hartmann, D., \& Mebold, U. 1998, A\&A, 332, 55

Rand, R. J. 1996, ApJ, 462, 712

Rand, R. J., \& Stone, J. M. 1996, AJ, 111, 190

Rand, R. J. 1997, in The Interstellar Medium in Galaxies, ed. J. M. van der Hulst (Dordrecht: Kluwer), 105

Reynolds, R. J. 1997, in The Physics of Galactic Halos, ed. H. Lesch, R.-J. Dettmar, U. Mebold, \& R. Schlickeiser (Berlin: Academie Verlag), 57

Santillan, A., Franco, J., Martos, M., \& Kim, J. 1999, ApJ, 515,657

Savage, B., Sembach, K. R., \& Lu, L. 1997, AJ, 113, 2158

Suchkov, A. A., Balsara, D. S., Heckman, T. M., Leitherner, C. 1994, ApJ, 430, 511

Swaters, R. A., Sancisi, R., \& van der Hulst, J. M. 1997, ApJ, 491, 140

Spitzer, L. 1956, ApJ, 124, 20

van der Hulst, T., \& Sancisi, R. 1988, AJ, 95, 1354 